\title{
The Relationship between Menopausal Original Article Symptoms and Heart Rate Variability in Middle Aged Women
}

\author{
Jin Oh Lee, Sung Goo Kang, Se Hong Kim, Seo Jin Park, Sang Wook Song*
}

Department of Family Medicine, St. Vincent's Hospital, The Catholic University of Korea School of Medicine, Suwon, Korea

Background: The study of the correlation of menopausal symptoms with heart rate variability (HRV) has not been adequate. The aim of this study was to investigate the relationship between postmenopausal symptoms measured by the menopause rating scale (MRS) and HRV.

Methods: We assessed postmenopausal symptoms (using MRS) with age, BMI, educational status, occupation, marital status, alcohol and caffeine consumption, smoking history, exercise, duration of sleep and amenorrhea, degree of anxiety and depression, menarcheal age, and heart rate variability. For evaluation of HRV, the record of electrocardiogram for 5 minutes in the resting state was divided into temporal categories and frequency categories, and analyzed.

Results: No significant differences in age, BMI, duration of amenorrhea, heart rate, systolic blood pressure, diastolic blood pressure, fasting blood sugar, triglyceride, and high-density lipoprotein were observed between two groups, which were divided according to menopausal symptoms. Low frequency/high frequency (LF/HF) ratio was significantly higher in symptomatic women, compared with asymptomatic women $(\mathrm{P}<0.05)$. No significant differences of HRV index by the severity of postmenopausal symptoms were observed. LF/HF ratio of HRV parameters showed a significant increase in moderate or severe degree of "hot flashes" and "sleep problem" score $(\mathrm{P}<0.05)$. Anxiety scale in symptomatic women was significantly higher than in asymptomatic women $(\mathrm{P}<0.05)$.

Conclusion: The above data suggest that postmenopausal symptoms are associated with altered autonomic control of heart rate. In particular, hot flashes and sleep problems in moderate or severe degree are related to increase of sympathetic nerve activity.

Keywords: Postmenopausal Symptoms; Menopause Rating Scale; Heart Rate Variability

\section{INTRODUCTION}

Received: August 30, 2010, Accepted: July 12, 2011

${ }^{*}$ Corresponding Author: Sang Wook Song

Tel: 82-31-249-7681, Fax: 82-31-248-7404

E-mail: sswkoj@unitel.co.kr

Korean Journal of Family Medicine

Copyright (C) 2011 The Korean Academy of Family Medicine

(c) This is an open-access article distributed under the terms of the Creative Commons Attribution Non-Commercial License (http://creativecommons.org/licenses/by-nc/3.0) which permits unrestricted noncommercial use, distribution, and reproduction in any medium, provided the original work is properly cited.
A woman spends $1 / 3$ of her life in the postmenopausal state. Health management during this period could be considered important because it affects the entire quality of life. ${ }^{1)}$ Menopause starts when menstruation is terminated permanently due to loss of ovary function, and its onset age is almost constant. ${ }^{2)}$ In Korea, the mean age of menopause is 48 years, and it occurs within 5-10 years around the age of 48 years. The menopausal state could be broadly divided into 3 stages: premenopause, perimenopause, and postmenopause. Based on the last menstrual period, if the 
period of amenorrhea is longer than 12 months, it is considered postmenopause. First 3 months of amenorrhea is considered premenopause. Perimenopause is amenorrhea of $3-12$ months, and the volume or frequency of menstruation becomes irregular due to estrogen deficiency during perimenopause. ${ }^{3)}$ Many women present with diverse physical as well as psychological symptoms during perimenopause and postmenopause. They are referred to as climacteric symptoms or menopausal symptoms.

According to the survey conducted by the Korean Society of Menopause, of 707 women who underwent natural menopause, $89 \%$ answered that they experienced menopausal symptoms or have symptoms currently. ${ }^{1)}$ Such menopausal symptoms include hot flashes, irregular heart beats, insomnia, and fatigue, in addition to myalgia and arthralgia, decreased desire, mood changes, hypersensitivity, anxiety, depression, memory impairment, etc., and urogenital symptoms, such as dry vagina, dyspareusia, cystitis, urodynia, and urinary urgency.

To estimate such climacteric symptoms, questionnaire survey methods, such as the Greene ${ }^{4)}$ menopause index, Kupperman index, and the menopause rating scale (MRS), etc. have been used. ${ }^{5)}$ Questionnaire survey methods have advantages in that they could be simply and readily used for assessment of menopausal symptoms.

The basic cause of menopausal symptoms is the complex relationship of estrogen metabolism and the autonomic nervous system. Therefore, imbalance of the autonomic nervous system may correlate with menopausal symptoms. Disharmony of the autonomic nervous system could be evaluated by measurement of heart rate variability (HRV) ${ }^{6}{ }^{6}$ Heart rate variability is cyclic interval changes of the heart rate. ${ }^{7)}$ This is the value that measures changes from one cardiac cycle to the next cycle or change of the RR interval with electrocardiogram. The activity level of the autonomic nervous system could be quantified by analysis of heart rate variability using power spectral analysis (time and frequency domain analysis). ${ }^{8)}$

Until now, study of the correlation of such menopausal symptoms with heart rate variability has not been adequate. In this study, the relationship of menopausal symptoms with heart rate variability was examined using a questionnaire survey method.

\section{METHODS}

\section{Study Subjects and Periods}

Among female patients who underwent physical examination at an university hospital or visited an outpatient clinic of the department of family from January 2010 to August 2010, this study was conducted on 51 patients whose amenorrhea was longer than 6 months, but less than 3 years. Of these, 15 patients were excluded because of following reasons: past experience of medications that may mediate an effect on HRB; hormone replacement therapy; total hysterectomy or bilateral oophrectomy; cases of thyroid disease; cases involving endocrine diseases such as diabetes, arrhythmia, cardiovascular disease; severe anemia with hemoglobin lower than $10 \mathrm{mg} / \mathrm{dL}$; and cases involving a history of psychological disease such as insomnia, depression, or schizophrenia. The final number of study subjects was 36.

\section{Study Methods}

1) The scale of menopausal symptoms

Data were collected by the subjects who agreed to participate in the study and filled out the questionnaire by themselves. Social and demographic characteristics of study subjects, and diseases currently under treatment, past surgical history, body mass index, education, profession, marital status, smoking, drinking, regular exercise, coffee drinking, sleeping hours, age of menarche, and the amenorrhea period were included in this questionnaire. In addition, for assessment of depression symptoms and anxiety symptoms, the hospital anxiety depression scale (HADS) was employed simultaneously. ${ }^{9)}$ The MRS Korean version, was used to measure climacteric symptoms. ${ }^{10)}$ The MRS has been used as an evaluation tool for screening of menopausal syndrome and treatments. This tool consists of items for total 11 symptoms, and previous diverse menopausal symptoms were divided into three categories: psychological domain, somato-vegetative domain, and urogenital domain. ${ }^{10)}$ Each question has a severity scale of symptoms as none ( 0 point), slight ( 1 point), moderate ( 2 points), severe ( 3 points), or very severe ( 4 points). The total point is the sum of the points of each question, and it is distributed from a minimum of 0 to a maximum of 44 points. 
Higher scores reflect severe levels of symptoms. The mean total score for Asians is 7.2 points, psychological symptoms is 2.9 points, physical symptoms is 3.3 points, and urogenital symptoms is 1.0 point. ${ }^{10)}$ In our study, 5 points of the MRS was considered as the cut-off point, and scores higher than 5 points were classified into the group with menopausal symptoms. In addition, in regard to the severity of menopausal symptoms, $0-4$ points were classified as the group with no/little symptoms, 5-8 points as the mild group, 9-16 points as the moderate group, and higher than 17 points as the severe group. ${ }^{10)}$

\section{2) Measurement of HRV}

To control the influence of circadian rhythm, HRV was measured in the fasting condition, after 10 minutes of sufficient rest, and between $8 \mathrm{AM}$ and $12 \mathrm{PM}$ using the SA-2000E (Medicore, Seoul, Korea). ${ }^{11)}$ Subjects were instructed not to drink alcohol on the day before measurement, and not to smoke on the day of measurement. For measurement, participants were seated on a chair, and an electrode was attached to the right wrist and the left ankle; measurement was performed for 5 minutes in the resting state.

For temporal analysis of the R-R interval change during a period of 5 minutes, we measuered the standard deviation of normal to normal intervals in milliseconds (SDNN), which simultaneously reflects the change caused by the parasympathetic system primarily and the square root of the mean squared differences of successive normal to normal intervals in milliseconds (RMSSD). As for the frequency category, the low frequency area (LF, $0.04-0.15 \mathrm{~Hz}$ ), the high frequency area (HF, $0.15-0.4 \mathrm{~Hz}$ ), and the LF/HF ratio were obtained, and statistically analyzed.

\section{3) Statistical analysis}

For statistical analysis, the SPSS ver. 12.0 (SPSS Inc., Chicago, IL, USA) was used. Regularity was examined using the ShapiroWilk test, and, if it followed the normal distribution, the score difference among the groups was analyzed by t-test. For cases that did not follow the normal distribution, significance was examined by Mann-Whitney test. For comparison of the symptom severity, the non-parametric test Kruskal-Wallis test was applied. In each analysis, a P-value of $<0.05$ was considered statistically significant.

\section{RESULTS}

\section{Characteristics of Study Subjects}

The final number of study subjects was 36 ; housewives were most prevalent, and all were married.

The Cronbach $\alpha$ of MRS was 0.841 . In the HADS, internal consistency of anxiety scale was 0.921 , and internal consistency of depression scale was 0.670 . According to the standard of MRS score, the group with menopausal symptoms included 26 patients (72.2\%), and the group without menopausal symptoms included 10 patients (27.8\%). The mean value of MRS index of the group with menopausal symptoms and the group without menopausal symptoms was 8.4 and 2.7, respectively. Age, body mass index, amount of coffee consumption, heart rate, blood pressure, fasting blood glucose, triglycerides, and HDL cholesterol value did not differ significantly. In the group with menopausal symptoms, sleep hours were less and anxiety trait was elevated in comparison with the group without menopausal symptoms $(\mathrm{P}<0.05)$ (Table $1)$.

\section{Association between the Presence or Absence and the Level of Menopausal Symptoms and HRV}

The LF/HF ratio of the group with menopausal symptoms was found to be significantly higher than that of the group without menopausal symptoms (Table 2).

To examine the difference of HRV according to the level of climacteric symptoms, they were compared using the nonparametric Kruksal-Wallis test. HRV according to the level of climacteric symptoms did not show significant differences (Table 3).

\section{Association between the Level of Hot Flashes and HRV}

Subjects were divided into two groups; those with a lowerthan-mild level of hot flashes, and the group with a higherthan-moderate level of hot flashes. According to the results comparing differences of HRV index, the LF/HF ratio was found to be elevated in the group whose hot flashes were higher than moderate, and other indexes of HRV between the two groups did not differ (Table 4). 
Jin Oh Lee, et al: The Relationship between Menopausal Symptoms and Heart Rate Variability in Middle Aged Women

Table 1. Baseline characteristics of subjects.

\begin{tabular}{lccc}
\hline \multicolumn{1}{c}{ Characteristics } & $\begin{array}{c}\text { Asymptomatic women (MRS }<5) \\
(\mathrm{n}=10)\end{array}$ & $\begin{array}{c}\text { Symptomatic women (MRS } \geq 5) \\
(\mathrm{n}=26)\end{array}$ & $\begin{array}{c}\text { P-value* } \\
\text { Age (y) }\end{array}$ \\
Body mass index $\left(\mathrm{kg} / \mathrm{m}^{2}\right)$ & $50.20 \pm 0.85$ & $51.42 \pm 0.49$ & $0.543^{\dagger}$ \\
Duration of amenorrhea (mo) & $25.48 \pm 4.17$ & $23.06 \pm 2.02$ & $0.108^{*}$ \\
Duration of sleep (h/d) & $22.20 \pm 3.69$ & $16.38 \pm 2.31$ & $0.209^{\dagger}$ \\
Coffee intake (cups/d) & $6.45 \pm 0.17$ & $5.87 \pm 0.18$ & $0.045^{\dagger}$ \\
Heart beat (beats/min) & $1.83 \pm 0.31$ & $1.41 \pm 0.23$ & $0.241^{\dagger}$ \\
Systolic blood pressure (mm Hg) & $64.20 \pm 6.07$ & $68.35 \pm 12.64$ & $0.330^{*}$ \\
Diastolic blood pressure (mm Hg) & $129.70 \pm 17.41$ & $107.04 \pm 33.19$ & $0.050^{*}$ \\
Fasting blood sugar (mg/dL) & $77.70 \pm 12.57$ & $77.96 \pm 16.26$ & $0.964^{*}$ \\
Triglyceride (mg/dL) & $91.60 \pm 6.58$ & $100.57 \pm 43.27$ & $0.523^{*}$ \\
High-density lipoprotein (mg/dL) & $132.40 \pm 11.22$ & $117.65 \pm 16.79$ & $0.057^{\dagger}$ \\
Anxiety score (HADS) & $55.60 \pm 9.48$ & $56.55 \pm 14.80$ & $0.855^{*}$ \\
Depression score (HADS) & $1.20 \pm 0.33$ & $4.38 \pm 0.64$ & $0.08 \pm 1.94$ \\
\hline
\end{tabular}

Values are presented as mean \pm SD.

MRS: menopause rating scale, HADS: hospital anxiety depression scale.

*P-values were obtained by t-test. ${ }^{\dagger} \mathrm{P}$-values were obtained by Mann-Whitney test.

Table 2. Heart rate variability of asymptomatic women vs. symptomatic women.

\begin{tabular}{lccc}
\hline \multicolumn{1}{c}{ Characteristics } & $\begin{array}{c}\text { Asymptomatic women (MRS }<5) \\
(\mathrm{n}=10)\end{array}$ & $\begin{array}{c}\text { Symptomatic women (MRS } \geq 5) \\
(\mathrm{n}=26)\end{array}$ & P-value* $^{*}$ \\
\hline SDNN (ms) & $31.83 \pm 4.17$ & $40.94 \pm 6.54$ & 0.411 \\
RMSSD (ms) & $31.44 \pm 4.96$ & $32.78 \pm 3.50$ & 0.837 \\
LF $\left(\mathrm{ms}^{2} ; 0.04-0.15 \mathrm{~Hz}\right)$ & $228.66 \pm 69.15$ & $231.88 \pm 66.57$ & $0.780^{\dagger}$ \\
HF $\left(\mathrm{ms}^{2} ; 0.15-0.04 \mathrm{~Hz}\right)$ & $226.24 \pm 59.67$ & $177.39 \pm 51.20$ & $0.270^{\dagger}$ \\
LF/HF ratio & $1.18 \pm 0.16$ & $2.45 \pm 0.52$ & 0.027 \\
\hline
\end{tabular}

Values are presented as mean $\pm \mathrm{SE}$.

SDNN: standard deviation of normal to normal intervals, RMSSD: square root of the mean squared differences of successive normal to normal intervals, LF/HF: low-frequency/high-frequency.

${ }^{*}$ P-values were obtained by t-test. ${ }^{\dagger} \mathrm{P}$-values were obtained by Mann-Whitney test.

\section{Association between the Level of Sleep notdiffer(Table 5). \\ Disorders and HRV}

As in hot flash symptoms analysis, subjects were divided into two groups; a lower-than-mild level of sleep disturbance, group and a higher-than-moderate level of sleep disturbance group. According to the results comparing differences of HRV index, the $\mathrm{LF} / \mathrm{HF}$ ratio in a higher-than-moderate level of sleep disturbance group showed statistically significant elevation. Other indexes did

\section{DISCUSSION}

Heart rate is determined by the proprietary autonomy of the sinoatrial node and the autonomic nervous system. The autonomic nervous system, which controls the sinoatrial node, 
Table 3. Heart rate variability according to severity of postmenopausal symptoms.

\begin{tabular}{lccccc}
\hline \multicolumn{1}{c}{ Variable } & No/little $(\mathrm{n}=10)$ & Mild $(\mathrm{n}=9)$ & Moderate $(\mathrm{n}=13)$ & Severe $(\mathrm{n}=4)$ & P-value* \\
\hline SDNN (ms) & $31.83 \pm 4.17$ & $27.78 \pm 2.36$ & $43.68 \pm 8.47$ & $61.61 \pm 32.70$ & 0.373 \\
RMSSD (ms) & $31.44 \pm 4.96$ & $22.92 \pm 1.20$ & $38.82 \pm 6.39$ & $35.34 \pm 3.00$ & 0.122 \\
LF $\left(\mathrm{ms}^{2} ; 0.04-0.15 \mathrm{~Hz}\right)$ & $228.66 \pm 69.15$ & $130.77 \pm 35.51$ & $316.85 \pm 127.06$ & $179.31 \pm 73.72$ & 0.725 \\
HF $\left(\mathrm{ms}^{2} ; 0.15-0.04 \mathrm{~Hz}\right)$ & $226.24 \pm 59.67$ & $77.78 \pm 17.35$ & $239.28 \pm 95.19$ & $190.93 \pm 94.88$ & 0.340 \\
LF/HF ratio & $1.18 \pm 0.16$ & $2.35 \pm 0.91$ & $2.65 \pm 0.82$ & $2.03 \pm 0.99$ & 0.670 \\
\hline
\end{tabular}

Values are presented as mean \pm SE.

SDNN: standard deviation of normal to normal intervals, RMSSD: square root of the mean squared differences of successive normal to normal intervals, LF/HF: low-frequency/high-frequency.

*P-values by Kruksal-Wallis test.

Table 4. Heart rate variability according to severity of hot flashes.

\begin{tabular}{lccc}
\hline \multicolumn{1}{c}{ Variable } & No or mild $(\mathrm{n}=21)$ & Moderate or severe $(\mathrm{n}=15)$ & P-value* $^{*}$ \\
\hline SDNN (ms) & $29.95 \pm 2.86$ & $50.23 \pm 10.45$ & 0.079 \\
RMSSD (ms) & $30.17 \pm 4.29$ & $35.54 \pm 3.23$ & 0.359 \\
LF (ms ${ }^{2}$ 0.04-0.15 Hz) & $177.31 \pm 37.13$ & $307.54 \pm 110.27$ & $0.522^{\dagger}$ \\
HF (ms ${ }^{2}$; 0.15-0.04 Hz) & $202.43 \pm 59.94$ & $176.51 \pm 47.66$ & $0.877^{\dagger}$ \\
LF/HF ratio & $1.41 \pm 0.21$ & $3.06 \pm 0.85$ & 0.034 \\
\hline
\end{tabular}

Values are presented as mean $\pm \mathrm{SE}$.

SDNN: standard deviation of normal to normal intervals, RMSSD: square root of the mean squared differences of successive normal to normal intervals, LF/HF: low-frequency/high-frequency.

*P-values were obtained by t-test. ${ }^{\dagger} \mathrm{P}$-values were obtained by Mann-Whitney test.

Table 5. Heart rate variability according to severity of sleep problems.

\begin{tabular}{lccc}
\hline \multicolumn{1}{c}{ Variable } & No or mild $(\mathrm{n}=21)$ & Moderate or severe $(\mathrm{n}=15)$ & P-value* $^{*}$ \\
\hline SDNN (ms) & $32.65 \pm 2.51$ & $46.46 \pm 11.05$ & 0.241 \\
RMSSD (ms) & $30.52 \pm 2.95$ & $35.05 \pm 5.50$ & 0.440 \\
LF $\left(\mathrm{ms}^{2} ; 0.04-0.15 \mathrm{~Hz}\right)$ & $209.23 \pm 48.78$ & $261.95 \pm 103.50$ & $0.769^{\dagger}$ \\
HF $\left(\mathrm{ms}^{2} ; 0.15-0.04 \mathrm{~Hz}\right)$ & $197.06 \pm 40.70$ & $184.18 \pm 79.61$ & $0.259^{\dagger}$ \\
LF/HF ratio & $1.29 \pm 0.19$ & $3.23 \pm 0.83$ & 0.037 \\
\hline
\end{tabular}

Values are presented as mean \pm SE.

SDNN: standard deviation of normal to normal intervals, RMSSD: square root of the mean squared differences of successive normal to normal intervals, LF/HF: low-frequency/high-frequency.

*P-values were obtained by t-test. ${ }^{\dagger} \mathrm{P}$-values were obtained by Mann-Whitney test.

is constantly influenced by diverse changes of environment, and the cyclic interval change of heart rate that appears at that time is referred to as HRV. ${ }^{12)}$ Analysis of such HRV, which could be used in assessing the activity level of the autonomic nervous system, could be performed relatively inexpensively; it is noninvasive and quantitative; thus, numerous studies have been conducted. Consequently, the task force of the European Society of Cardiology and the North American Society of Pacing and 
Electrophysiology standardized the method for measurement of HRV and its physiological and clinical uses in $1996 .{ }^{13)}$ Clinical application of LF is controversial; nonetheless, LF has been used as the index of control of the sinoatrial node of the heart by the sympathetic nerve, and HF as the index of control of the sinoatrial by the vagus nerve. And the LF/HF ratio, which reflects activity of sympathetic nerves, has been used as the index of the balance state of the autonomic nervous system. ${ }^{14)}$ It has been reported that the general characteristic of HRV is shown in general by time domain analysis; nonetheless, frequency domain analysis better shows the balance state of the sympathetic nerve and the parasympathetic nerve. ${ }^{12)}$

In this study, the relationship of menopausal symptoms and HRV was examined. In the menopausal group, the LF/HF ratio was found to be significantly elevated in comparison with the group without menopausal symptoms. Until now, studies comparing the HRV of young premenopausal women with that of postmenopausal women have reported that the LF/HF ratio was elevated in postmenopausal women. ${ }^{14)}$ However, studies comparing HRV of the group with menopausal symptoms and the group without menopausal symptoms in perimenopausal and postmenopausal woman, such as our research, have not been conducted. Results of this study have shown that the group with menopausal symptoms showed a greater increase in sympathetic nerve activity.

No significant differences of HRV index were observed according to severity of postmenopausal symptoms. Results of this study do not concur with results of a study reported by Ahn et al. in 2005, ${ }^{15)}$ which applied HRV as a standard of climacteric symptoms. They reported that in the group with severe menopausal symptoms, the SDNN and the RMSSD were reduced. Nevertheless, the study by Ahn et al. ${ }^{15)}$ has a limitation in that the number of subjects was small $(n=16)$. Our study also has a small number of subjects; thus, it is difficult to draw a concrete conclusion in regard to the association of the level of menopausal symptoms with HRV. Studies with larger number of subjects will be required in the future.

According to our results that show the association of the 11 subcategories of the MRS and the index of HRV, the LF/HF ratio was found to increase in the group whose levels of hot flashes and sleep disorders were higher than moderate. The results concur with results reported by Hoikkala et al. ${ }^{16)}$ in 2009 that showed the normalized LF and normalized HF value were different by the level of hot flashes. In addition, although direct comparison is difficult, the findings are similar to those of the study by Rebecca et al. in 2009, which was on hot flashes and control of the heart by the vagus nerve and showed that HF decreased when hot flashes were severe. Other studies and ours found that, according to hot flash symptoms, one of which is menopausal symptoms, imbalance of the autonomic nervous system such as reduction of the activity level of the parasympathetic nerve may be present. The mechanism of postmenopausal sleep disorders has not been elucidated. However, several studies speculate that hot flashes induce wakefulness during sleep, resulting in chronic sleep deprivation and fatigue. ${ }^{17)}$ Consequently, deterioration of the sleep quality lowers the level of activity of the parasympathetic nerves. ${ }^{12)}$ When the autonomic nervous system, which maintains the electrical stability of heart, is impaired, arrhythmia or consequent death may increase. ${ }^{12)}$ Therefore, our study found that the level of imbalance of the autonomic nervous system was different by the severity of hot flashes or sleep disorder symptoms.

The group with menopausal symptoms had higher anxiety traits with statistical significance that the group without menopausal symptoms. When postmenopausal physical symptoms manifest, anyone experiences negative self-esteem such as hurt pride, uselessness, lack of self-confidence, etc.; thus, a state of emotional crisis, such as anxiety, depression, etc. may be induced. ${ }^{18)}$

This study has several limitations. First, although 5-minute measurement of HRV has been applied as a useful tool for evaluation of the autonomic nervous system function, which controls the heart, the area of very LF, which directly shows the level of activity of the sympathetic nervous system, could be measured with accuracy using 24-hour measurement. ${ }^{13)}$ So, our study measured SDNN and the LF area among measurements of 5-minute HRV; then attempted indirect analysis. For more accurate studies, measurement of 24-hour HRV is required. Second, the number of subjects was not sufficient; thus, the association of the level of menopausal symptoms with HRV was analyzed using a non-parametric testing method. It is suggested that studies on a larger number of subjects should be conducted.

Despite such limitations, our study observed that the presence or absence of menopausal symptoms was associated with HRV. The $\mathrm{LH} / \mathrm{HF}$ ratio became different depending on the severity 
of hot flashes and sleep disorders, and the elevation of anxiety trait and other emotional disorders were observed in the group with menopausal symptoms. The association of menopausal symptoms with cardiovascular disease could not be directly compared only by HRV; nonetheless, in cases experiencing a higher-than-moderate level of hot flashes and sleep disorders, based on the increase of the LF/HF ratio, the increased level of activity of the sympathetic nervous system could be confirmed in our study.

\section{REFERENCES}

1. Kim MJ, Kim JH. How long do menopausal hot flushes really last? J Korean Soc Menopause 2009; 15:73-8.

2. Speroff L, Glass RH, Kase NG. Clinical gynecologic endocrinology and infertility. 5th ed. Baltimore: Williams \& Wilkins; 1994.

3. McKinlay SM, Brambilla DJ, Posner JG. The normal menopause transition. Maturitas 1992;14:103-15.

4. Greene JG. A factor analytic study of climacteric symptoms. J Psychosom Res 1976;20:425-30.

5. Heinemann K, Ruebig A, Potthoff P, Schneider HP, Strelow F, Heinemann LA, et al. The Menopause Rating Scale (MRS) scale: a methodological review. Health Qual Life Outcomes 2004;2:45.

6. Earnest CP, Lavie CJ, Blair SN, Church TS. Heart rate variability characteristics in sedentary postmenopausal women following six months of exercise training: the DREW study. PLoS One 2008;3:e2288.

7. Lee YJ, Kim MS, Kim BT, Kwak TH, Shim JY, Lee HR. Heart rate variability in metabolic syndrome. J Korean Acad Fam Med 2002;23:1432-9.

8. Pomeranz B, Macaulay RJ, Caudill MA, Kutz I, Adam D, Gordon D, et al. Assessment of autonomic function in humans by heart rate spectral analysis. Am J Physiol
1985;248:H151-3.

9. Herrmann C. International experiences with the Hospital Anxiety and Depression Scale--a review of validation data and clinical results. J Psychosom Res 1997;42:17-41.

10. Berlin Center for Epidemiology and Health Research. MRSthe menopause rating scale [Internet]. Berlin: ZEG Berlin; [cited 2009 Jun 20]. Available from: http://www.menopauserating-scale.info.

11. Choi CJ, Choi WS, Kim KS. The changes in heart rate variability between morning and afternoon. J Korean Acad Fam Med 2008;29:579-84.

12. Seo AR, Kang SG, Shin JH, Song SW. The relationship between sleep quality and heart rate variability in middleaged men. Korean J Health Promot Dis Prev 2009;9:289-95.

13. Heart rate variability. Standards of measurement, physiological interpretation, and clinical use. Task Force of the European Society of Cardiology and the North American Society of Pacing and Electrophysiology. Eur Heart J 1996; 17:354-81.

14. Neves VF, Silva de Sa MF, Gallo L Jr, Catai AM, Martins LE, Crescencio JC, et al. Autonomic modulation of heart rate of young and postmenopausal women undergoing estrogen therapy. Braz J Med Biol Res 2007;40:491-9.

15. Ahn SJ, Hwang JH, Choi JE, Cho JH, Jang JB, Lee KS. Practical use of HRV as barometer of climacteric symptom. J Orient Obstet Gynecol 2005;18:192-202.

16. Hoikkala H, Haapalahti P, Viitasalo M, Vaananen H, Sovijarvi AR, Ylikorkala $\mathrm{O}$, et al. Association between vasomotor hot flashes and heart rate variability in recently postmenopausal women. Menopause 2010;17:315-20.

17. Lim WJ. Sleep in menopause. Sleep Med Psychophysiol 2002;9:96-9.

18. Beck CT. The occurrence of depression in women and the effect of the women's movement. J Psychiatr Nurs Ment Health Serv 1979;17:14-6. 